\title{
AUTOMORPHIC CUSP FORMS CONSTRUCTED FROM THE WEIL REPRESENTATION
}

\author{
BY S. RALLIS AND G. SCHIFFMANN
}

Communicated by J. A. Wolf, September 10, 1976

We recall the notation and results of [3].

Let $\mathbf{Q}$ be the rational numbers.

We let $L$ be a $Q$ integral lattice in $Q^{k}$, i.e. $Q\left(\xi_{1}, \xi_{2}\right) \in \mathbf{Z}$ for all $\xi_{1}, \xi_{2} \in$ $L$. Let $L_{*}(Q)$ be the $Q$ dual of $L$, i.e. $L_{*}(Q)=\left\{\eta \in \mathbf{R}^{k} \mid Q(\eta, \xi) \in \mathbf{Z}, \forall \xi \in L\right\}$. Then $L_{*}(Q) / L$ is a finite Abelian group, and we let $N_{L}$ be the exponent of $L_{*}(Q) / L$, i.e. the smallest positive integer $x$ so that $x \cdot \xi \in L$ for all $\xi \in L_{*}(Q)$. Choosing a Z-basis $X_{i}$ of $L$, we let $D_{Q(L)}=\operatorname{det}\left\{Q\left(X_{i}, X_{j}\right)\right\}$. Then the integer $D_{Q(L)}$ is independent of the choice of basis of $L$.

Then we define

$$
\Gamma_{L}(Q)=\{g \in O(Q) \mid g(L)=L\}
$$

and

$$
\begin{aligned}
\Gamma^{L}(Q)=\left\{\left(\left[\begin{array}{ll}
a & b \\
c & d
\end{array}\right], \epsilon\right) \mid a, b, c, d \in \mathbf{Z}, a d-b c=1,\right. \\
\left.b \equiv 0 \bmod 2 \text { and } c \equiv 0 \bmod 2 N_{L}\right\} .
\end{aligned}
$$

Then $\Gamma_{L}(Q)$ is an arithmetic subgroup of $O(Q)$ and $\Gamma^{L}(Q) /($ cyclic group of order 4) is an arithmetic subgroup of $\mathrm{PSl}_{2}(\mathbf{R})$ (contained in the $\Gamma_{\vartheta}$ theta group). Then using the corollary to Theorem 5 of [3] we have

THEOREM 1. Let $\varphi$ be a $\widetilde{K} \times K$ finite function in $\mathrm{F}_{Q}^{+}\left(s^{2}-2 s\right)$ with $s>$ $1 / 2 k$. Then the sum with $(G, g) \in \widetilde{\mathrm{SI}_{2}} \times O(Q)$,

$$
T_{\varphi}^{L}(G, g)=\sum_{\xi \in L} \pi_{Q}(G, g)^{-1}(\varphi)(\xi),
$$

is absolutely convergent. Moreover, for $(\Omega, \gamma) \in \Gamma^{L}(Q) \times \Gamma_{L}(Q)$, we have the functional equation

$$
T_{\varphi}^{L}(G \Omega, g \gamma)=\sigma_{Q}^{L}(\Omega, \gamma) T_{\varphi}^{L}(G, g),
$$

where $\sigma_{Q}^{L}$ is a unitary character on $\Gamma^{L}(Q) \times \Gamma_{L}(Q)$ taking values in $S_{4}$ (where $S_{j}=\left\{z \in \mathrm{C} \mid z^{j}=1\right\}$ for $j$ any positive integer). Moreover, $T_{\varphi}^{L}$ is a $C^{\infty}$ function on $\widetilde{\mathrm{ST}_{2}} \times O(Q)$ satisfying $D * T_{\varphi}^{L}(G, g)=T_{\pi}^{L}{ }_{Q^{(D) \varphi}}(G, g)$ for any $D$ in the

AMS (MOS) subject classifications (1970). Primary 10D20. 
universal enveloping algebra of $\widetilde{\mathrm{ST}_{2}} \times O(Q)(*$ represents differentiation on the left). In particular, $\omega_{\mathrm{Sl}_{2}} * T_{\varphi}^{L}=\left(s^{2}-2 s\right) T_{\varphi}^{L}$. Finally we have the estimate

$$
\left|T_{\varphi}^{L}(G, g)\right| \leqslant M r_{G}^{s-1 / 2}\left\|g^{-1}\right\|_{k}^{s+k / 2-2}
$$

where $M$ is some positive constant independent of $(G, g), r_{G}$ denotes the $A$ part of $G$ in the Iwasawa decomposition of $G=K_{G} a\left(r_{G}\right) n\left(x_{G}\right)$, and \|\|$_{k}$ denotes the Frobenius norm of a linear operator on $\mathbf{R}^{k}$.

REMARK 1. The function $T_{\varphi}^{L}$ is an automorphic form on $\widetilde{\mathrm{SI}_{2}} \times O(Q)$ in the sense of the definitions in [1].

REMARK 2. The unitary character $\sigma_{Q}^{L}$ on $\Gamma^{L}(Q) \times \Gamma_{L}(Q)$ is given as $\sigma_{Q}^{L}(\Omega, \omega)=c(\Omega)$, where the map $\Omega m c(\Omega)$ on $\Gamma^{L}(Q)$ is given by

$$
c\left(\left[\begin{array}{ll}
\alpha & \beta \\
\gamma & \delta
\end{array}\right], \epsilon\right)=(\operatorname{sgn} \epsilon)^{k} b_{\delta}^{k}\left(\frac{2 \gamma}{\delta}\right)^{k}\left(\frac{D_{Q(L)}}{\delta}\right)
$$

where $\gamma \neq 0$ with

$$
b_{\delta}=\left\{\begin{aligned}
1 & \text { if } \delta \equiv 1 \bmod 4, \\
\sqrt{-1} & \text { if } \delta \equiv 3 \bmod 4
\end{aligned}\right.
$$

and ( $\rightarrow$ ) the quadratic residue symbol as given in [4].

Using Remark 2 we then construct on $P=\{z \in \mathrm{C} \mid \operatorname{Im}(z)>0\}$, the upper half plane, a half-integral multiplier system for the discrete arithmetic group

$$
\Delta_{N_{L}}=\left\{\left[\begin{array}{ll}
\alpha & \beta \\
\gamma & \delta
\end{array}\right] \in \mathrm{Sl}_{2}(\mathrm{Z}) \mid \gamma \equiv 0 \bmod 2 N_{L}, \beta \equiv 0 \bmod 2\right\}
$$

of degree $s$, taking values in $S_{4}$. That is: if $v_{Q}(G)=\{c((G, 1))\}^{-1} \psi_{2}(G)$ with

$$
\psi_{2}(G)= \begin{cases}1 & \text { if } c_{G} \neq 0, \\ \operatorname{sgn}\left(d_{G}\right) & \text { if } c_{G}=0\end{cases}
$$

then

$$
v_{Q}\left(G_{1} G_{2}\right)\left(c_{3} z+d_{3}\right)^{s}=v_{Q}\left(G_{1}\right) v_{Q}\left(G_{2}\right)\left(c_{1} z+d_{1}\right)^{s}\left(c_{2} z+d_{2}\right)^{s},
$$

where $G_{1}, G_{2} \in \Delta_{N_{L}}$ with

$$
G_{i}=\left[\begin{array}{ll}
a_{i} & b_{i} \\
c_{i} & d_{i}
\end{array}\right] \text { and } G_{1} G_{2}=\left[\begin{array}{ll}
a_{3} & b_{3} \\
c_{3} & d_{3}
\end{array}\right]
$$

(where $z^{s}=|z|^{s} e^{\sqrt{-1}(\arg z) s}$ with $-\pi<\arg z \leqslant \pi$ ).

Then using Theorem 1 and the corollary to Theorem 5 of [3], we deduce the following. 
THEOREM 2. Let $\varphi$ be a function belonging to $E_{Q}\left(s^{2}-2 s, s, s_{1}, 0\right)$ (with $s>1 / 2 k$ and $s_{1}=s-1 / 2(a-b)$ ) of the form on $\Omega_{+}: \varphi(X)=$

$$
Q(X, X)^{s-1} e^{-\pi Q(X, X)}\left\|X_{+}\right\|^{-\left(s+s_{1}+k / 2-2\right)} Q\left(X, \xi_{+}\right)^{s_{1}}
$$

where $\xi_{+} \in \mathbf{C}^{a}$ is a nonzero complex isotropic vector, i.e. $Q\left(\xi_{+}, \xi_{+}\right)=0$. Then we let

$$
\widetilde{T}_{\varphi}^{L}(z, g)=(\operatorname{Im} z)^{s / 2} T_{\varphi}^{L}\left(\left(\left[\begin{array}{ll}
x & y \\
0 & x^{-1}
\end{array}\right], 1\right), g\right)
$$

with $z=-y / x+\sqrt{-1} x^{2} \in P$. Then we have the expansion

$$
\widetilde{T}_{\varphi}^{L}(z, g)=\sum_{n \in \mathrm{Z} ; n \geqslant 1} n^{s-1} e^{\pi \sqrt{-1} z n} \varphi_{n}^{s_{1}}(g)
$$

where

$$
\varphi_{n}^{s_{1}}(g)=\sum_{\{M \in L \mid Q(M, M)=n\}} Q\left(M, g^{-1} \xi_{+}\right)^{s_{1}}\left\|(g M)_{+}\right\|^{-\left(s+s_{1}+k / 2-2\right)}
$$

Then $\mathrm{T}_{\varphi}^{L}(z, g)$ is an antiholomorphic cusp form in $z$ for $\Delta_{N_{L}}$ of degree $|s|$ form for $\Delta_{N_{L}}$ with multiplier $v_{Q}$ of degree $s$, that is

$$
\widetilde{T}_{\varphi}^{L}\left(\frac{a z+b}{c z+d}, g\right)=v_{Q}(G)(c z+d)^{s} \widetilde{T}_{\varphi}^{L}(z, g)
$$

with $G=\left[\begin{array}{ll}a & b \\ c & d\end{array}\right] \in \Delta_{N_{L}}$. Moreover, we have that $\widetilde{T}_{\varphi}^{L}(z, g)$ is a cusp form in $z$ for $\Delta_{N_{L}}$, that is, $\widetilde{T}_{\varphi}^{L}$ is holomorphic at $\infty$ (from 1.5) and $T_{\varphi}^{L}(u+\sqrt{ }-\overline{1} v, g)=$ $O\left(v^{-(s-1 / 4)}\right)$ as $v \rightarrow 0$ uniformly in $u$.

REMARK 3. Choosing the quadratic form $Q=x y+z w$ on $\mathbf{R}^{4}$ and a suitable $Q$ integral lattice $L \subseteq \mathbf{R}^{4}$, we obtain from the construction above automorphic forms similar to $\Omega\left(\tau_{1}, \tau_{2}, z\right)$ in [6]. We also note the construction of related automorphic forms in [2] and [5] for the case $k=3$.

REMARK 4. From the invariance of $\widetilde{T}_{\varphi}^{L}$ in the $O(Q)$ variable relative to $\Gamma_{L}(Q)$, we see that $\varphi_{n}^{s_{1}}(g \gamma)=\varphi_{n}^{s_{1}}(g)$ for all $g \in O(Q), \gamma \in \Gamma^{L}(Q)$. The interpretation of formula (1.5) for $\widetilde{T}_{\varphi}^{L}$ is simply the Fourier expansion of $\tilde{T}_{\varphi}^{L}$ at $\infty$ with each Fourier coefficient $\varphi_{n}^{s_{1}}(g) n^{s-1}$ an automrophic form for $O(Q)$ relative to $\Gamma_{L}(Q)$.

REMARK 5. In a manner similar to the construction above (with the added assumption that $b=2)$, we start with the function $\varphi \in E_{Q}\left(s^{2}+2 s, s, 0, s_{2}\right) \subseteq$

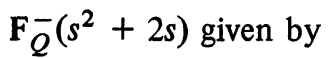

$$
\varphi(Y)=|Q(Y, Y)|^{|s|-1} e^{\pi Q(Y, Y)} Q\left(X, \xi_{-}\right)^{-s_{2}} \text { on } \Omega_{-}
$$

where $s<-1 / 2 k$ and $s_{2}=|s|+1 / 2 a-1$ and $\xi_{-} \in \mathbf{C}^{b}$, nonzero complex isotropic, i.e. $Q\left(\xi_{-}, \xi_{-}\right)=0$. Then as in Theorem 2 we let 


$$
\widetilde{T}_{\varphi}^{L}(z, g)=\sum_{n \in Z ; n \leqslant-1}|n|^{|s|-1} e^{\pi \sqrt{-1} n z} \widetilde{\varphi}_{n}^{s_{2}}(g)
$$

where

$z \in \bar{P}=$ lower half plane.

$$
\tilde{\varphi}_{n}^{s_{2}}(g)=\sum_{\{M \in L \mid Q(M, M)=n\}} Q\left(M, g^{-1} \xi_{-}\right)^{-s_{2}},
$$

Then $\mathbf{T}_{\varphi}^{L}(z, g)$ is an antiholomorphic cusp form in $z$ for $\Delta_{N_{L}}$ of degree $|s|$ with multiplier $v_{Q}$.

Then we can analyze the cuspidal behavior of each $\widetilde{\varphi}_{n}^{s_{2}}$ determined in Remark 5.

THEOREM 3. Let $b=2$ and let $\tilde{\varphi}_{n}^{s_{2}}$ be as in Remark 5. Then for the unipotent radical $H$ of any rational maximal parabolic subgroup of $O(Q)$ we have

$$
\int_{H / H \cap \Gamma_{L}(Q)} \tilde{\varphi}_{n}^{s_{2}}(g h) d h \equiv 0
$$

(with dh an $H$ invariant measure on $H / H \cap \Gamma_{L}(Q)$ ) for all $g \in O(Q)$ and all $n \leqslant-1$.

REMARK 6. Theorem 3 implies that the family of automorphic forms $\widetilde{\varphi}_{n}^{s_{2}}$ belongs to the space of cusp forms (in the sense of [1]) of $L^{2}\left(O(Q) / \Gamma_{L}(Q)\right)$.

The case $b=2$ turns out to be critical in the proof of Theorem 3. The basic idea behind the proof of Theorem 3 is what we call the Cusp Vanishing Theorem.

THEOREM 4. Let $\varphi \in \mathbf{F}_{Q}^{-}\left(s^{2}+2 s\right)$ be a $\widetilde{K} \times K$ finite function with $b=$ 2 and $s<-1 / 2 k$. Then for any $X \in \Omega_{-}$and for the unipotent radical $H$ of any rational maximal parabolic subgroup of $O(Q), \int_{H / H} X \varphi(g h(X)) d \mu_{x}(h) \equiv 0$ for all $g \in O(Q)$ (with $d \mu_{x}$ some $H$ invariant measure on $H / H^{X}, H^{X}=$ isotropy group of $X)$.

Again we note the importance of the case $b=2$. If $b=2$, then $O(Q) / K$ is a Hermitian symmetric space. We let $F=f+p$ be the Cartan decomposition of the Lie algebra of $O(Q)$. Then we have the direct sum $F_{\mathrm{C}}=\mathfrak{f}_{\mathrm{C}} \oplus p^{+} \oplus$ $p^{-}$, where $p^{-}$and $p^{+}$span the holomorphic and antiholomorphic tangent vectors at the "origin" in $O(Q) / K$. Then we recall the construction of a family of holomorphic discrete series representations of $O(Q)$. We consider $K=$ $O(a) \times O(2)$, and let $\chi_{n}: K \rightarrow S^{1}$ be the unitary character on $K$ which is trivial on $O(a)$ and maps

$$
O(2)=\left\{\left[\begin{array}{cc}
\cos \theta & \sin \theta \\
-\sin \theta & \cos \theta
\end{array}\right] 1-\pi<\theta \leqslant \pi\right\}
$$


to $e^{\sqrt{ }-1 n \theta}(n \in Z)$. Then we form the "holomorphic" unitarily induced representation space $H\left(O(Q) / k, \chi_{n}\right)=\left\{\varphi: O(Q) \rightarrow \mathrm{C} \mid \varphi(g k)=\varphi(g) \chi_{n}(k)\right.$ for all $g \in O(Q), k \in K, \varphi * W \equiv 0$ for all $W \in p^{+}$, and $\left.\int_{O(Q) / K}|\varphi(g)|^{2} d \sigma(g)<\infty\right\}$ with $* W$, convolution on the left and $d \sigma$ some $O(Q)$ invariant measure on $O(Q) / K$. Then we have

THEOREM 5. The representation on $O(Q)$ in $A_{s}^{-}$(see Remark 1 in [3]) is equivalent to the "holomorphic" induced representation of $O(Q)$ in $H\left(O(Q) / K, \chi_{s_{2}}\right)$ where $s_{2}=|s|+1 / 2 a-1$.

REMARK 7. The representation of $O(Q)$ in $A_{s}^{-}$(for $b=2$ ) is thus always "square integrable". Moreover $A_{s}^{-}$is "integrable" if $s<2-k$.

Corollary to THeOREM 5. Let $s<2-k$. Then each $\tilde{\varphi}_{n}^{s_{2}}$ given in Remark 5 is a "Poincaré series" on $O(Q) / \Gamma_{L}(Q)$. That is, there exists a $K$ finite function $q_{n} \in H\left(O(Q) / K, \chi_{s_{2}}\right)$ so that $\tilde{\varphi}_{n}(g)=\Sigma_{\gamma \in \Gamma_{L}(Q)} q_{n}\left(\gamma g^{-1}\right)$.

\section{BIBLIOGRAPHY}

1. Harish-Chandra, Automorphic forms on semisimple Lie groups, Lecture Notes in Math., vol. 62, Springer-Verlag, Berlin and New York, 1968. MR 38 \#1216.

2. S. Niwa, Modular forms of half integral weight and the integral of certain theta. functions, Nagoya Math. J. 56 (1975), 147-161. MR 51 \#361.

3. S. Rallis and G. Schiffmann, Discrete spectrum of the Weil representation, Bull. Amer. Math. Soc. 83 (1977), 267-270.

4. G. Shimura, On modular forms of half integral weight, Ann. of Math. (2) 97 (1973), 440-481. MR 48 \#10989.

5. T. Shintani, On construction of holomorphic cusp forms of half integral weight, Nagoya Math. J. 58 (1975), 83-136.

6. D. Zagier, Modular forms associated to real quadratic fields Invent. Math. 30 (1975), $1-48$.

DEPARTMENT OF MATHEMATICS, UNIVERSITY OF NOTRE DAME, NOTRE DAME, INDIANA 46556

INSTITUTE DE RECHERCHE MATHÉMATIQUE AVANCÉE, UNIVERSITÉ LOUIS PASTEUR, 7, RUE RENÉ DESCARTES, 67084-STRASBOURG, CEDEX, FRANCE (Current address of G. Schiffmann)

Current address (Stephen Rallis): Department of Mathematics, Princeton University, Princeton, New Jersey 08540 